\title{
About Pascal's tetrahedron with hypercomplex entries
}

\author{
C. Cruz ${ }^{*}$ M. I. Falcão*,† and H. R. Malonek*,** \\ ${ }^{*}$ Center for Research and Development in Mathematics and Applications, University of Aveiro, Portugal \\ ${ }^{\dagger}$ Department of Mathematics and Applications, University of Minho, Portugal \\ ** Department of Mathematics, University of Aveiro, Portugal
}

\begin{abstract}
It is evident, that the properties of monogenic polynomials in $(n+1)$-real variables significantly depend on the generators $e_{1}, e_{2}, \ldots, e_{n}$ of the underlying $2^{n}$-dimensional Clifford algebra $C \ell_{0, n}$ over $\mathbb{R}$ and their interactions under multiplication. The case of $n=3$ is studied through the consideration of Pascal's tetrahedron with hypercomplex entries as special case of the general Pascal simplex for arbitrary $n$, which represents a useful geometric arrangement of all possible products. The different layers $\mathscr{L}_{k}$ of Pascal's tetrahedron (or pyramid) are built by ordered symmetric products contained in the trinomial expansion of $\left(e_{1}+e_{2}+e_{3}\right)^{k}, k=0,1, \ldots$
\end{abstract}

Keywords: Pascal's tetrahedron, Clifford Analysis

PACS: 02.30.-f, 02.30.Lt, 02.10.Ox

\section{INTRODUCTION}

The following formulae (ㅁ) and (B]) are examples of the role that complex (imaginary) entries can play when used in powers of binomials. Since we have

$$
(1+i)^{4 l+1}=(1+i)^{4 l}(1+i)=(2 i)^{2 l}(1+i)=(-4)^{l}(1+i) l=1,2, \ldots,
$$

the binomial expansion of the left side of $(\mathbb{I})$ implies immediately the validity of two binomial identities

$$
\left(\begin{array}{c}
4 l+1 \\
0
\end{array}\right)-\left(\begin{array}{c}
4 l+1 \\
2
\end{array}\right)+\left(\begin{array}{c}
4 l+1 \\
4
\end{array}\right)-\cdots+\left(\begin{array}{c}
4 l+1 \\
4 l
\end{array}\right)=(-4)^{l}
$$

and

$$
\left(\begin{array}{c}
4 l+1 \\
1
\end{array}\right)-\left(\begin{array}{c}
4 l+1 \\
3
\end{array}\right)+\left(\begin{array}{c}
4 l+1 \\
5
\end{array}\right)-\cdots-\left(\begin{array}{c}
4 l+1 \\
4 l+1
\end{array}\right)=(-4)^{l}
$$

after separation of the real and imaginary part.

Working in hypercomplex analysis with $n$ different non-commutative imaginary units, the following general question seems natural: What is changing in the ordinary $n$-dimensional arrangement of multinomial coefficients (Pascal's simplex) if the real entries are substituted by $n$ imaginary units $e_{1}, e_{2}, \ldots, e_{n}$ ?

The fact that the structure of the layer in Pascal's simplex rules the composition of a special set of Appell polynomials in terms of hypercomplex variables was already mentioned in the paper [I]. Due to the relevance of the answer for applications in Clifford analysis, we consider the set $\left\{e_{1}, e_{2}, \ldots, e_{n}\right\}$ as being an orthonormal basis of the Euclidean vector space $\mathbb{R}^{n}$ with a non-commutative product according to the multiplication rules

$$
e_{k} e_{l}+e_{l} e_{k}=-2 \delta_{k l}, k, l=1, \ldots, n,
$$

where $\delta_{k l}$ is the Kronecker symbol. Then the set $\left\{e_{A}: A \subseteq\{1, \ldots, n\}\right\}$ with $e_{A}=e_{h_{1}} e_{h_{2}} \ldots e_{h_{r}}, 1 \leq h_{1}<\cdots<$ $h_{r} \leq n, e_{\emptyset}=e_{0}=1$, forms a basis of the $2^{n}$-dimensional Clifford algebra $C \ell_{0, n}$ over $\mathbb{R}$. We embed $\mathbb{R}^{n+1}$ in $C \ell_{0, n}$ by identifying $\left(x_{0}, x_{1}, \ldots, x_{n}\right) \in \mathbb{R}^{n+1}$ with $x=x_{0}+\underline{x} \in \mathscr{A}:=\operatorname{span}_{\mathbb{R}}\left\{1, e_{1}, \ldots, e_{n}\right\} \subset C \ell_{0, n}$. Here $x_{0}=\operatorname{Sc}(x)$ and $\underline{x}=\operatorname{Vec}(x)=e_{1} x_{1}+\cdots+e_{n} x_{n}$ are the so-called scalar resp. vector part of the paravector $x \in \mathscr{A}$. The conjugate of $x$ is given by $\bar{x}=x_{0}-\underline{x}$ and its norm by $|x|=(x \bar{x})^{\frac{1}{2}}=\left(x_{0}^{2}+x_{1}^{2}+\cdots+x_{n}^{2}\right)^{\frac{1}{2}}$. Obviously, we can identify the case $n=1$ with the complex algebra case by $i:=e_{1}$. 


\section{PASCAL'S TETRAHEDRON WITH HYPERCOMPLEX ENTRIES}

As mentioned in the beginning, the consideration of an arbitrary value of $n$ leads to an arrangement of multinomial coefficients following the multinomial expansion theorem. But essential and non trivial effects of the non-commutative multiplication can already be seen in the case of $n=3$. The corresponding 3-simplex is the Pascal's tetrahedron (see [2]) with hypercomplex entries. The different layers $\mathscr{L}_{k}$ of it are built by the elements of the trinomial expansion of $\left(e_{1}+e_{2}+e_{3}\right)^{k}, k=0,1, \ldots$ As example, let us consider the case $k=3$, i.e. the construction of the third layer $\mathscr{L}_{3}$. By taking into account non-commutativity the expansion can explicitly be written in the following order:

$$
\begin{aligned}
\left(e_{1}+e_{2}+e_{3}\right)^{3}= & e_{1}^{3} \\
+ & \left(e_{1} e_{1} e_{2}+e_{1} e_{2} e_{1}+e_{2} e_{1} e_{1}\right)+\left(e_{1} e_{1} e_{3}+e_{1} e_{3} e_{1}+e_{3} e_{1} e_{1}\right) \\
+ & \left(e_{1} e_{2} e_{2}+e_{2} e_{1} e_{2}+e_{2} e_{2} e_{1}\right) \\
& \quad+\left(e_{1} e_{2} e_{3}+e_{1} e_{3} e_{2}+e_{2} e_{1} e_{3}+e_{2} e_{3} e_{1}+e_{3} e_{1} e_{2}+e_{3} e_{2} e_{1}\right)+\left(e_{1} e_{3} e_{3}+e_{3} e_{1} e_{3}+e_{3} e_{3} e_{1}\right) \\
& +e_{2}^{3}+\left(e_{2} e_{2} e_{3}+e_{2} e_{3} e_{2}+e_{3} e_{2} e_{2}\right)+\left(e_{2} e_{3} e_{3}+e_{3} e_{2} e_{3}+e_{3} e_{3} e_{2}\right)+e_{3}^{3} .
\end{aligned}
$$

This expansion corresponds to the case $k=3$ in Pascal's tetrahedron for real entries, given in the general form (cf. [2])

$$
(a+b+c)^{k}=\sum_{m=0}^{k} \sum_{s=0}^{m}\left(\begin{array}{c}
k \\
m
\end{array}\right)\left(\begin{array}{c}
m \\
s
\end{array}\right) a^{k-m} b^{m-s} c^{s}, \quad a, b, c \in \mathbb{R} .
$$

The corresponding layer $\mathscr{L}_{3}$ written in ordered rows as arrangement of the different monomials corresponding to the increasing row-index $m^{\mathbb{\square}}$ has the form ${ }^{\square}$ :

$$
\begin{aligned}
& m=0 \\
& \left(\begin{array}{l}
3 \\
0
\end{array}\right) a^{3} \\
& m=1 \\
& m=2 \\
& \left(\begin{array}{l}
3 \\
2
\end{array}\right)\left(\begin{array}{l}
2 \\
0
\end{array}\right) a b^{2} \\
& \left(\begin{array}{l}
3 \\
1
\end{array}\right)\left(\begin{array}{l}
1 \\
0
\end{array}\right) a^{2} b \\
& m=3 \\
& \left(\begin{array}{l}
3 \\
3
\end{array}\right)\left(\begin{array}{l}
3 \\
0
\end{array}\right) b^{3} \\
& \left(\begin{array}{l}
3 \\
3
\end{array}\right)\left(\begin{array}{l}
3 \\
1
\end{array}\right) b^{2} c \\
& \left(\begin{array}{l}
3 \\
1
\end{array}\right)\left(\begin{array}{l}
1 \\
1
\end{array}\right) a^{2} c \\
& \left(\begin{array}{l}
3 \\
2
\end{array}\right)\left(\begin{array}{l}
2 \\
2
\end{array}\right) a c^{2} \\
& \left(\begin{array}{l}
3 \\
3
\end{array}\right)\left(\begin{array}{l}
3 \\
2
\end{array}\right) b c^{2}
\end{aligned}
$$

The differences between $\left(e_{1}+e_{2}+e_{3}\right)^{3}$ and $(a+b+c)^{3}$ are obvious and due to the non-commutativity of the hypercomplex imaginary units we cannot obtain (వ) by substituting $a=e_{1}, b=e_{2}, c=e_{3}$ in (面). Nevertheless, it exists a way to describe the trinomial expansion of $\left(e_{1}+e_{2}+e_{3}\right)^{k}$ formally in the same way as that of $(a+b+c)^{3}$. To do so one has to use the following (cf. [3])

Definition 1 (Symmetric Product) Let $V_{+}$, be a commutative or non-commutative ring, $a_{k} \in V, k=1, \ldots, n$, then the " $\times$ "-product is defined by

$$
a_{1} \times a_{2} \times \cdots \times a_{n}=\frac{1}{n !} \sum_{\pi\left(s_{1}, \ldots, s_{n}\right)} a_{s_{1}} a_{s_{2}} \ldots a_{s_{n}}
$$

where the sum runs over all permutations of all $\left(s_{1}, \ldots, s_{n}\right)$.

together with the

Convention: If the factor $a_{j}$ occurs $\mu_{j}$-times in (पI), we briefly write

$$
\underbrace{a_{1} \times \cdots \times a_{1}}_{\mu_{1}} \times \cdots \times \underbrace{a_{n} \times \cdots \times a_{n}}_{\mu_{n}}=a_{1}{ }^{\mu_{1}} \times a_{2}{ }^{\mu_{2}} \times \cdots \times{a_{n}}^{\mu_{n}}
$$

and set parentheses if the powers are understood in the ordinary way.

\footnotetext{
${ }^{1}$ The index $s$ is increasing in NW-SE diagonal direction, whereas $(m-s)$ increases in NE-SW diagonal direction. Since $\left(\begin{array}{c}k \\ m\end{array}\right)\left(\begin{array}{c}m \\ s\end{array}\right)=\left(\begin{array}{c}k \\ m\end{array}\right)\left(\begin{array}{c}m \\ m-s\end{array}\right)=$ $\left(\begin{array}{c}k \\ k-m\end{array}\right)\left(\begin{array}{c}m \\ s\end{array}\right)$ all elements with the same corresponding index in this three directions are the same (symmetry property).

2 To be exact, Pascal's tetrahedron includes only the trinomial coefficients $\left(\begin{array}{l}k \\ m\end{array}\right)\left(\begin{array}{c}m \\ s\end{array}\right)$. This is the case if $a=b=c=1$.
} 
Consequently, the multinomial theorem for entries of a commutative or non-commutative ring, $V$ is obtained in the same form as the ordinary multinomial theorem for real entries (cf. [3]).

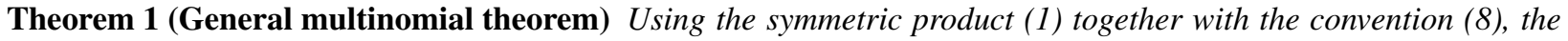
powers of a sum of $n$ different elements $a_{1}, \ldots a_{n}$ of an arbitrary commutative or non-commutative ring $V$ can be expanded in the form

$$
\left(a_{1}+a_{2}+\cdots+a_{n}\right)^{k}=\sum_{|\mu|=k}\left(\begin{array}{l}
k \\
\mu
\end{array}\right) \vec{a}^{\mu}
$$

where, as usual, $\left(\begin{array}{l}k \\ \mu\end{array}\right)=\frac{k !}{\mu !},|\mu|=\mu_{1}+\mu_{2}+\cdots+\mu_{n}$ and $\vec{a}^{\mu}=a_{1}{ }^{\mu_{1}} \times a_{2}{ }^{\mu_{2}} \times \cdots \times a_{n}{ }^{\mu_{n}}, k \in \mathbb{N}$.

It follows straightforward that the trinomial expansion (5) can now be rewritten as

$$
\left(e_{1}+e_{2}+e_{3}\right)^{k}=\sum_{m=0}^{k} \sum_{s=0}^{m}\left(\begin{array}{c}
k \\
m
\end{array}\right)\left(\begin{array}{c}
m \\
s
\end{array}\right) e_{1}^{k-m} \times e_{2}^{m-s} \times e_{3}^{s} .
$$

From (4) it follows, for example, that the central element in the layer $\mathscr{L}_{3}$ of Pascal's tetrahedron is given by $\left(\begin{array}{l}3 \\ 2\end{array}\right)\left(\begin{array}{l}2 \\ 1\end{array}\right) e_{1} \times e_{2} \times e_{3}=\left(e_{1}\left[e_{2} e_{3}+e_{3} e_{2}\right]+e_{2}\left[e_{1} e_{3}+e_{3} e_{1}\right]+e_{3}\left[e_{1} e_{2}+e_{2} e_{1}\right]\right)=0$ and the final form of $\mathscr{L}_{3}$ is

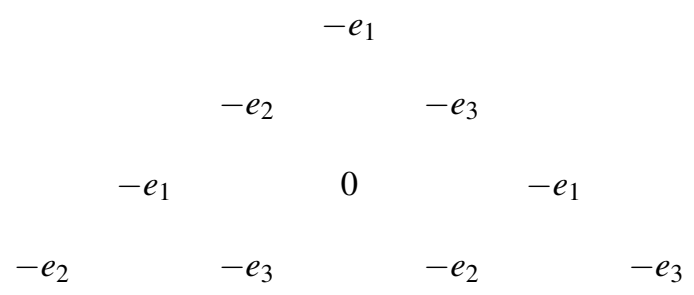

\section{THE COMPLETE CHARACTERIZATION OF PASCAL'S HYPERCOMPLEX TETRAHEDRON}

For the complete characterization of Pascal's tetrahedron built from hypercomplex entries for arbitrary values of $k$ we use the fact that $\left(e_{1}+e_{2}+e_{3}\right)$ is a paravector and therefore $\left(e_{1}+e_{2}+e_{3}\right)^{2 l}=(-1)^{l}(1+1+1)^{l}, l=0,1,2, \ldots$ This means that in the case of even $k(k=2 l)$ the $\mathscr{L}_{k}$ is filled with the multinomial numbers of $(1+1+1)^{l}$ multiplied by $(-1)^{l}$, but the rows, (NE-SW)- resp. (NW-SE)-diagonals with odd indices contain only zeros. For the case of odd $k(k=2 l+1)$ we use the fact that $\left(e_{1}+e_{2}+e_{3}\right)^{2 l+1}=(-1)^{l}(1+1+1)^{l}\left(e_{1}+e_{2}+e_{3}\right)$ which shows (without any calculation of the concrete value of $\left.e_{1}^{k-m} \times e_{2}^{m-s} \times e_{3}^{s}\right)$ that a layer of odd degree contains only real multiples of the hypercomplex generators $e_{1}$ or $e_{2}$ or $e_{3}$, with the exception of $k=1$. Taking this into account we can prove the following

Theorem 2 Let $\mathscr{E}_{(k, m, s)}^{3}:=\left(\begin{array}{c}k \\ m\end{array}\right)\left(\begin{array}{c}m \\ s\end{array}\right) e_{1}^{k-m} \times e_{2}^{m-s} \times e_{3}^{s}, k=0,1,2, \ldots ; m=0,1, \ldots, k ; s=0,1, \ldots, m$ and

$$
\left(e_{1}+e_{2}+e_{3}\right)^{k}=\sum_{m=0}^{k} \sum_{s=0}^{m}\left(\begin{array}{c}
k \\
m
\end{array}\right)\left(\begin{array}{c}
m \\
s
\end{array}\right) e_{1}^{k-m} \times e_{2}^{m-s} \times e_{3}^{s}=\sum_{m=0}^{k} \sum_{s=0}^{m} \mathscr{E}_{(k, m, s)}
$$

Then the entries $\mathscr{E}_{(k, m, s)}$ of Pascal's hypercomplex tetrahedron are given in the following form:

I. If $k$ is even then

$$
\mathscr{E}_{(k, m, s)}= \begin{cases}(-1)^{\frac{k}{2}}\left(\begin{array}{c}
\frac{k}{2} \\
\frac{m}{2}
\end{array}\right)\left(\begin{array}{l}
\frac{m}{2} \\
\frac{s}{2}
\end{array}\right), & \text { m even, s even } \\
0, & \text { otherwise. }\end{cases}
$$




\section{If $k$ is odd then}

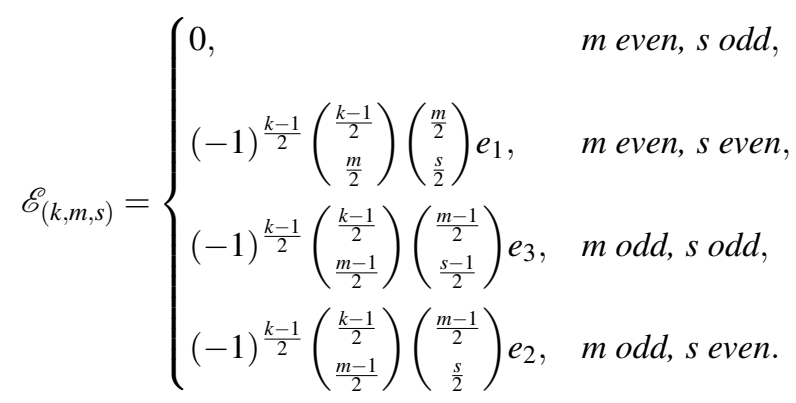

Moreover, following the recurrence properties of Pascal's tetrahedron with real entries (cf. [2]) it can be shown that the numbers on every $k$-th layer are the sum of the three adjacent numbers in the $(k-1)$-th layer (the layer above the $k$-th layer in the tetrahedron), each one multiplied by $e_{1}$ or $e_{2}$ or $e_{3}$, respectively, i. e. we have

$$
\mathscr{E}_{(k, m, s)}=\mathscr{E}_{(k-1, m-1, s-1)} e_{3}+\mathscr{E}_{(k-1, m-1, s)} e_{2}+\mathscr{E}_{(k-1, m, s)} e_{1} .
$$

The following pictures try to illustrate Pascal's tetrahedron as well as the mentioned recurrence relation for the case $k=4$.
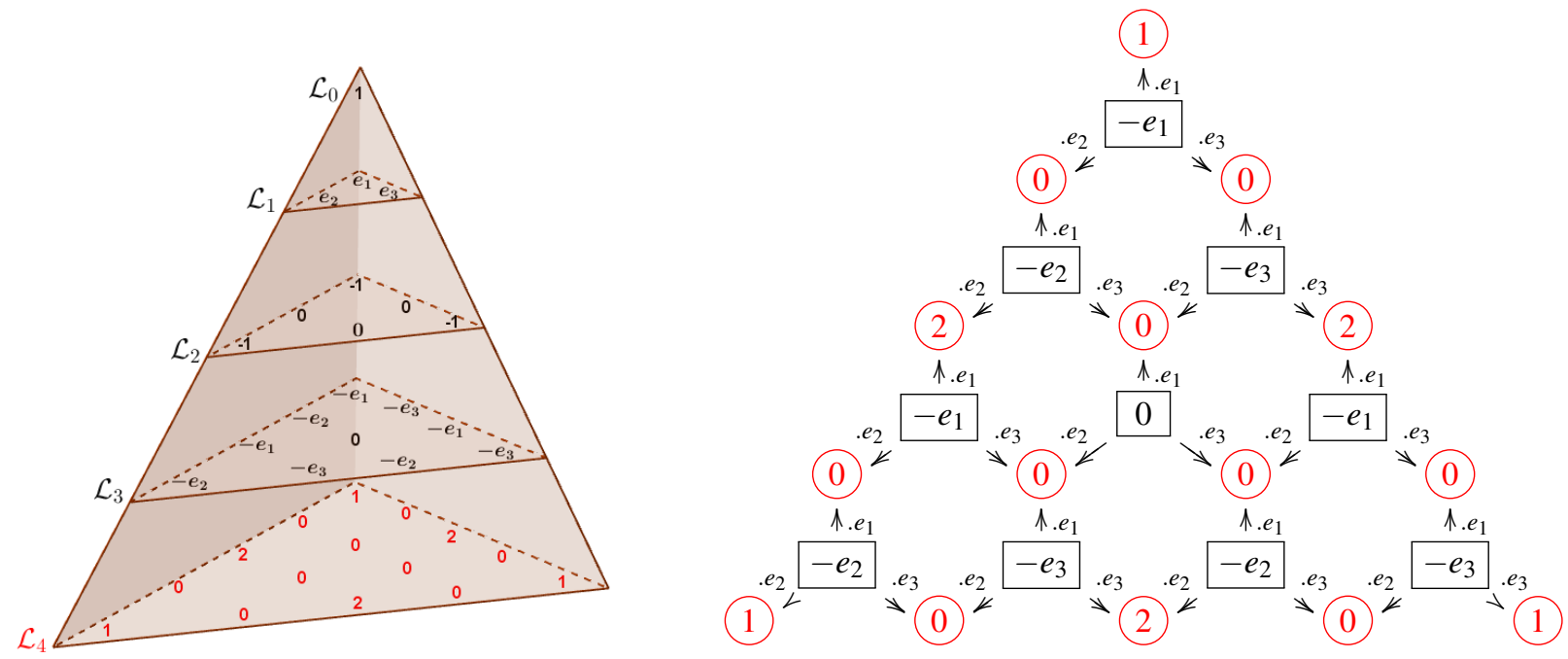

\section{ACKNOWLEDGMENTS}

This work was supported by FEDER founds through COMPETE-Operational Programme Factors of Competitiveness ("Programa Operacional Factores de Competitividade") and by Portuguese funds through the Center for Research and Development in Mathematics and Applications (University of Aveiro) and the Portuguese Foundation for Science and Technology ("FCT-Fundação para a Ciência e a Tecnologia"), within project PEst-C/MAT/UI4106/2011 with COMPETE number FCOMP-01-0124-FEDER-022690. The research of the first author was also supported by FCT under the fellowship SFRH/BD/44999/2008.

\section{REFERENCES}

1. M. I. Falcão, and H. Malonek, "Clifford Analysis between Continuous and Discrete," in Numerical Analysis and Applied Mathematics, ed. by T. E. Simos et al., AIP Conference Proceedings 1048, American Institute of Physics, New York, 2008, pp. 682-685.

2. Boris A. Bondarenko, Generalized Pascal triangles and pyramids, their fractals, graphs, and applications. Transl. from the Russian by Richard C. Bollinger. (English) Santa Clara, CA: The Fibonacci Association, vii, 253 p. (1993).

3. H. R. Malonek, "Selected topics in hypercomplex function theory", in Clifford algebras and potential theory (ed. S.-L. Eriksson), Report 7, University of Joensuu, 2004, pp. 111-150. 